\title{
Mercados municipales, escenarios de saberes ancestrales y turismo en la ciudad de Quito-Ecuador. @()(0)
}

\author{
Municipal markets, scenarios of ancestral knowledge and tourism in the city \\ of Quito-Ecuador.
}

Ing. Jenny Elizabeth Suárez Velasco Mgtr. ${ }^{1}$, Ing. Gustavo Vladimir Paladines Mgtr. ${ }^{2}$, Dra. C. Maité Echarri Chávez. ${ }^{3}$ \& Dr. C. Eros Salinas Chávez. ${ }^{4}$

Recibido: 07-02-2019 / Revisado: 08-02-2019 /Aceptado: 05-03-2019/ Publicado: 04-04-2019

\begin{abstract}
.
DOI: https://doi.org/10.33262/cienciadigital.v9i2.375

Tourism constitutes a growing activity globally. Visiting distant and different geographical places becomes more and more common throughout the world. And there is a diversity of attractions that are promoted with greater force such as adventure, health or culture. The latter represents an interesting reason to travel to another place and experience the wealth of other peoples. Part of the culture is crafts, gastronomy, food, way of speaking, traditions, ancestral knowledge, art, etc.

In this context, based on the fact that municipal markets are a space for the expression of culture and ancestral knowledge that, beyond gastronomy, offer varied services and products that show the idiosyncrasy and tradition of the people, the present investigation constituted a effort to analyze the participation of these spaces of minority commerce, within the cultural tourism of the city of Quito.

As part of this qualitative research, several information and analysis documents were reviewed, to show how tourism is in Quito, how are the markets, how ancestral knowledge is considered, what is the gastronomic offer in the city, issues that served as basis for the study.

After presenting and analyzing the issues addressed, it was concluded that the municipal markets have an impact on the cultural tourism of the city, which are places that should improve, although the authorities have begun to make changes in some points such as hygiene and security. However, the attraction of the municipal markets
\end{abstract}

\footnotetext{
${ }^{1}$ Universidad de las Fuerzas Armadas, Sangolquí, Ecuador, jesuarez1@ @espe.edu.ec

${ }^{2}$ Universidad de las Fuerzas Armadas, Sangolquí, Ecuador, gvpaladines@ espe.edu.ec

${ }^{3}$ Universidad de La Habana, Cuba, mecharri@ftur.uh.cu

${ }^{4}$ Universidad de La Habana, Cuba, esalinas@ftur.uh.cu
} 
is often the informality of how it is traded, the personalized attention, the differentiated offer of other places of sale and gastronomy.

In addition, the need to take care of the local culture, ancestral knowledge and gastronomy as part of the expression of the people of Quito was highlighted, to avoid that those traits that manifest in a unique way the cultural heritage, are lost with the arrival of new ways of do things, often brought with globalization.

for the company that would be the investor.

Keywords: Municipal markets, Ancestral knowledge, Cultural tourism

\section{Resumen.}

El turismo constituye una creciente actividad a nivel global. El visitar lugares geográficos distantes y diferentes se vuelva cada vez más usual en todo el mundo. Y existe una diversidad de atractivos que se promocionan con mayor fuerza como los de aventura, salud o cultura. Este último representa un interesante motivo para viajar a otro lugar y poder vivenciar la riqueza de otros pueblos. Parte de la cultura son las artesanías, gastronomía, alimentos, forma de hablar, tradiciones, saberes ancestrales, arte, etc.

En ese contexto, partiendo del hecho de que los mercados municipales constituyen un espacio de expresión de cultura y saberes ancestrales que, más allá de la gastronomía, ofrecen variados servicios y productos que muestran la idiosincrasia y tradición de los pueblos, la presente investigación constituyó un esfuerzo para analizar la participación de estos espacios de comercio minoritario, dentro del turismo cultural de la ciudad de Quito. Como parte de esta investigación cualitativa, se revisaron varios documentos de información y análisis, para evidenciar cómo está el turismo en Quito, cómo están los mercados, cómo se consideran los saberes ancestrales, cómo es la oferta gastronómica en la urbe, temas que sirvieron de base para el estudio.

Luego de presentar y analizar los temas abordados, se pudo concluir que los mercados municipales tienen un impacto en el turismo cultural de la ciudad, que son lugares que deben mejorar, a pesar de que las autoridades han empezado a efectuar cambios en algunos puntos como higiene y seguridad. Sin embargo, el atractivo de los mercados municipales es muchas veces la informalidad de cómo se comercia, la atención personalizada, la oferta diferenciada de otros lugares de expendio y gastronomía.

Además, se destacó la necesidad de cuidar la cultura local, los saberes ancestrales y la gastronomía como parte de la expresión del pueblo quiteño, para evitar que aquellos rasgos que manifiestan de manera única el acervo cultural, se pierdan con la llegada de nuevas maneras de hacer las cosas, muchas veces traídas con la globalización.

Palabras claves: Mercados municipales, Saberes ancestrales, Turismo cultural 


\section{Introducción.}

La Capital del Ecuador fue fundada en 1534 por los españoles con el nombre de Quito; sin embargo, la ciudad ya existió desde hace unos diez mil años antes, según el sociólogo Salazar, quien afirma que estuvo habitada por indígenas y fue hasta 1532 "la última capital de los incas". En el contexto actual, la urbe capitalina fue la primera ciudad en ser declarada como "Patrimonio Cultural de la Humanidad" por la Organización de las Naciones Unidas para la Educación, la Ciencia y la Cultura (UNESCO), evento que sucedió en septiembre de 1978, debido principalmente a las construcciones coloniales de su sector central. Pero la urbe sigue manteniendo sus características en buen estado, ya que: "Tiene uno de los centros históricos mejores conservados de América Latina”. (Romero, 2018)

Quito es una ciudad que presenta características que la hacen única, como el estar rodeada de volcanes y cortada por la línea ecuatorial que divide los dos hemisferios (norte y sur). Además de estas cualidades, su colonialismo resalta en el casco central de la ciudad y dentro de estos atractivos pueden mencionarse algunos como el Panecillo, por su estatua tan reconocida de la Virgen, así como por la vista panorámica de la urbe desde ese punto; la Iglesia de la Compañía de Jesús que es una de las obras de arte barroco más destacadas del continente, la Iglesia de la Catedral, entre otros. También sobresalen otros atractivos más modernos de la capital, como el monumento a la Mitad del Mundo que se ubica en el sector norte, la denominada "Capilla del Hombre" construida por Oswaldo Guayasamín y la capilla que se ubica a los pies de la elevación denominada "Ruco Pichincha". (Macipe, s.f.)

En ese contexto, la ciudad de Quito representa uno de los lugares preferidos como destino turístico a nivel regional, e incluso nacional, a pesar de que existe en Ecuador una gran variedad de atractivos naturales, empezando la lista por las Islas Galápagos, las provincias del oriente, las playas, la sierra centro, norte y sur.

Al ser Quito un atractivo turístico, Pastor (2003) señala que “...el turismo siempre ha tenido un aspecto cultural", porque desde hace mucho tiempo, uno de los motivos para visitar otros países y ciudades, ha sido justamente el componente cultural de esos destinos, entre los que se cuentan infraestructura, manifestaciones históricas, artísticas o aquellas que generan atractivo por considerarse exóticas.

Los turistas culturales se pueden clasificar en 3 tipos que son: los especialistas, los motivados y los ocasionales. El primer conjunto incluye aquellos turistas que gustan de visitar y conocer otros lugares, porque coinciden con viajes de negocios; este grupo abarca entre $10 \%$ y $15 \%$ del turismo cultural. El segundo implica entre un $30 \%$ a $40 \%$ del total y se refiere a quienes se enfocan en conocer culturas de otros lugares; mientras que el tercero, que representa entre un $45 \%$ y $60 \%$, se compone de personas que viajan de manera irregular, puede ser por vacaciones esporádicas, para disfrutar de atractivos de otros países. (Pastor, 2003) 
Dentro del aspecto cultural se puede afirmar que "La cocina es cultura", como lo señala Julio Pazos, investigador de la gastronomía quiteña, quien sostiene que la "cocina ancestral ecuatoriana, en general, y capitalina, en particular, se ubica en los períodos cuando los pueblos prehispánicos empezaron a crear utensilios de cerámica”. (El Telégrafo, 2014)

En efecto, la alimentación es una parte fundamental de la cultura de cada pueblo; y trae consigo una serie de elementos tradicionales que han sido heredados de manera informal de padres a hijos, de abuelos a nietos o de tíos a sobrinos. A pesar de que la cultura nacional ha sido invadida por diferentes tradiciones de otros pueblos, incluyendo los colonizadores en el siglo XVI, se han conservado algunos rasgos y platillos de los indígenas que habitaban antes, como por ejemplo el choclo cocinado y la chicha de jora (El Telégrafo, 2014).

\section{Mercados municipales}

Los mercados municipales son espacios en los que se efectúan transacciones minoristas, para la compra de productos perecederos. Estos lugares representan un "pilar fundamental" para la adquisición de alimentos frescos por parte de los consumidores. En la actualidad, este tipo de formato comercial también utiliza la "estrategia de elasticidad cruzada", como ocurre en otros lugares comerciales, en la que por temporadas incrementan unos productos y bajan otros, lo que también permite la colocación de la producción agropecuaria de estacionalidad. (Martín, 2010)

De acuerdo a la información del Municipio de Quito, existen 30 mercados municipales en la urbe capitalina: 7 en el norte, 12 en el centro y 11 en el sur, como se observa en la Tabla N. 1.

Tabla 1. Mercados Municipales de Quito

\begin{tabular}{lll}
\hline Sector Norte & Sector Centro & Sector Sur \\
\hline Santa Clara & Ipiales & Santa Lucía \\
Iñaquito (La Carolina) & El Tejar & Los Andes \\
La Kennedy & Hermano Miguel & Chiriyacu \\
Andalucía & Arenas & La Ferroviaria \\
Cotocollao & San Juan & Villa Flora \\
Rumiñahui & San Roque & La Magdalena \\
Comité del Pueblo & América & Solanda \\
& La Vicentina & Quito Sur \\
& La Floresta & El Calzado \\
& Av. Pichincha & Santa Martha \\
& Central & La Mena \\
& San Francisco & \\
\hline
\end{tabular}

Fuente: (Guerrero, 2015, pág. 20)

\section{Saberes ancestrales}


Los saberes ancestrales representan aquellos conocimientos "que poseen los pueblos indígenas y comunidades, transmitidos de generación en generación y que no forman parte de la educación formal" (ACTIVATE, 2014). En ese sentido, todas aquellas enseñanzas y tradiciones que han sido pasadas de padres a hijos pueden constituirse como saberes ancestrales, siempre que no se hayan incluido dentro de los currículos de la educación que se recibe en escuelas, colegios o universidades.

Para Carvallo (2015), estos conocimientos han sido relegados y mal vistos por la sociedad, ya que se han considerado como supersticiones, o "mero folklore", y no se les dio el lugar e importancia de representar el pensar, sentir y experiencia de nuestros antepasados. Sin embargo, a partir de 2.001, año en que se publicó la declaración universal sobre la Diversidad Cultural por parte de la UNESCO, en la que se reconoce a los saberes ancestrales como "recursos para toda la humanidad", estos conocimientos se han comenzado a tomar como parte del patrimonio cultural de los pueblos.

El Ecuador es uno de los países latinoamericanos que “....reconocen los saberes ancestrales como componentes importantes para el desarrollo, y proponen valorar e incluir la diversidad cultural en este ámbito." En ese contexto, estos conocimientos aportan a la equidad de las comunidades en la configuración de los saberes sociales. (Pérez, 2018)

\section{Materiales y Métodos}

El presente estudio ha recopilado algunos datos sobre la ciudad de Quito, el turismo, los mercados municipales y los saberes ancestrales, para conocer la participación de los espacios de comercio minoritario, que representan escenarios de saberes ancestrales, en el turismo de la urbe capitalina.

Para esto, la investigación tuvo un enfoque cualitativo, ya que no se centró solamente en la estadística (Ramírez, 2011, pág. 31), sino que tomó en consideración otros estudios relativos al aspecto objeto de estudio, que son los mercados de la ciudad Quito, en relación con el turismo de los últimos años.

Además, se puede definir a la investigación como histórica y documental. En el primer caso, por cuanto el estudio ha considerado una serie de información de sucesos pasados respecto de lo que representan los mercados y la situación del turismo durante los últimos años en la ciudad Quito. También se puede definir a la investigación como documental, ya que tomó en cuenta varios artículos, estudios, revistas o informes, que contienen datos referentes al tema abordado. (Bernal, 2010, págs. 110-111)

Como métodos de investigación científica utilizados, se nombran al analítico y al sintético; ya que en primera instancia se procedió a descomponer la información consultada sobre la temática objeto de estudio, para entender cada una de sus partes por separado, y luego se integraron las respetivas observaciones, para consolidar los datos de tal manera que, se pudo 
llegar a determinar cómo influyen los mercados municipales al turismo de la ciudad capital del Ecuador. (Rodríguez, 2005, pág. 30)

\section{Resultados.}

Con el fin de una mejor comprensión de los resultados, se presentan algunos puntos de manera independiente, para luego consolidar los análisis en el apartado de la discusión, y también confrontar esto con otros estudios que tienen relación con la temática abordada.

\section{Datos de turismo de Ecuador}

En un contexto general, de acuerdo a datos de la Organización Mundial de Turismo (OMT), se puede verificar un incremento de $35 \%$ en la cantidad de visitantes que ingresó al país entre 2010 y 2016 (1.047.000 y 1.418 .000 respectivamente). En cambio, la diferencia entre 2016 y 2017 fue de 13,4\% aproximadamente, con lo que el número de turistas para ese año fue de 1.608.000. La variación de los ingresos estimados en dólares para el país por estos visitantes, se pueden verificar en la Figura No.1.

Figura 1. Ingresos de turistas en Ecuador en últimos años

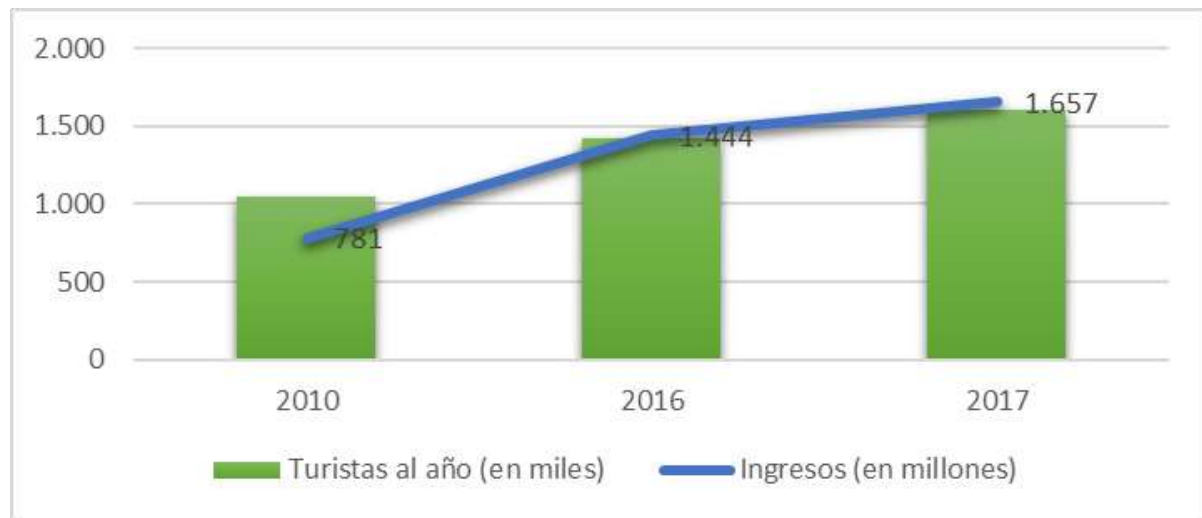

Fuente: (OMT, 2019, pág. 19)

\section{Estadísticas de turismo en Quito}

Quito ha recibido un porcentaje visitantes creciente durante los últimos años, excepto en el año 2016, en el que se tuvo una baja del 12\% respeto a 2015. Si bien casi todos los últimos años se registra incremento, 2013 y 2014 fueron los períodos de mayor impacto, como se revisa en la Tabla No. 2, elaborada en función de datos del Sistema Institucional de Indicadores Turísticos del Municipio de Quito (2019). 
Tabla 2. Llegadas de turistas a Quito últimos años

\begin{tabular}{ccc}
\hline Años & Turistas al año & \% Variación anual \\
\hline 2010 & 474.221 & $2,7 \%$ \\
2011 & 487.378 & $2,8 \%$ \\
2012 & 533.458 & $9,5 \%$ \\
2013 & 628.958 & $17,9 \%$ \\
2014 & 703.046 & $11,8 \%$ \\
2015 & 712.877 & $1,4 \%$ \\
2016 & 627.626 & $-12,0 \%$ \\
2017 & 652.931 & $4,0 \%$ \\
2018 (estimado) & 692.492 & $6,1 \%$ \\
\hline
\end{tabular}

Fuente: (SIIT, 2019)

Aparte de aquellos ecuatorianos "no residentes" que llegan a Quito, Estados Unidos es el país de donde provienen la mayoría de turistas extranjeros, seguido no muy de cerca por Colombia, España, México y otros, como se observa en la siguiente información del Municipio de Quito. Mientras que Norteamérica aporta con un 24,4\% de visitantes, Europa alcanzó 13,2\% en el año 2017. De países orientales como China y Japón apenas se registró el 2,3\% de acuerdo a la información del Municipio de Quito que consta en la Tabla No.3.

Tabla 3. Llegadas de turistas a Quito por origen en año 2017

\begin{tabular}{lcc}
\hline \multicolumn{1}{c}{ Origen } & $\begin{array}{c}\text { Turistas al año } \\
\mathbf{2 0 1 7}\end{array}$ & \% del total \\
\hline Ecuador & 151.725 & $21,9 \%$ \\
Estados Unidos & 148.742 & $21,5 \%$ \\
Colombia & 55.306 & $8,0 \%$ \\
España & 32.785 & $4,7 \%$ \\
México & 20.593 & $3,0 \%$ \\
Alemania & 20.411 & $2,9 \%$ \\
Canadá & 19.901 & $2,9 \%$ \\
Perú & 17.438 & $2,5 \%$ \\
Reino Unido & 17.351 & $2,5 \%$ \\
Resto del mundo & 208.240 & $30,1 \%$ \\
Total & $\mathbf{6 9 2 . 4 9 2}$ & $\mathbf{1 0 0 , 0 \%}$ \\
\hline
\end{tabular}

Fuente: (SIIT, 2019)

Dado que la capital es un centro cultural de festividades y celebraciones, también hay turistas que viajan desde el extranjero para fechas específicas como las que constan en la Tabla No. 4, en la que se presentan datos comparativos de los dos últimos años. 
Tabla 4. Turistas que llegaron a Quito en festividades en años 2017 y 2018

\begin{tabular}{lccc}
\hline \multicolumn{1}{c}{ Fecha } & Turistas 2017 & Turistas 2018 & Variación \\
\hline Carnaval (feb/mar) & 5.443 & 7.575 & $39,2 \%$ \\
Semana santa (mar/abr) & 5.100 & 4.967 & $-2,6 \%$ \\
Día trabajo (mayo) & 6.121 & 6.167 & $0,8 \%$ \\
Batalla Pichincha (mayo) & 5.239 & 5.372 & $2,5 \%$ \\
Independencia (ago) & 7.025 & 7.175 & $2,1 \%$ \\
Día difuntos (nov) & 6.110 & 6.354 & $4,0 \%$ \\
Fiestas Quito (dic) & 10.220 & 10.629 & $4,0 \%$ \\
Total & $\mathbf{4 5 . 2 5 8}$ & $\mathbf{4 8 . 2 3 9}$ & $\mathbf{6 , 6 \%}$ \\
\hline
\end{tabular}

Fuente: (SIIT, 2019)

Se observa que la festividad en la que más visitantes acuden a Quito, es en diciembre para sus fiestas de fundación; sin embargo, el crecimiento más alto entre 2017 y 2018 se observa que ocurrió en el feriado de carnaval que suele festejarse en febrero o marzo de cada año (39\% para los años revisados).

Vacaciones, ocio y recreación fueron los motivos principales de la visita de extranjeros a Quito según datos de agosto del 2015. Entre otros motivos constan la visita a familiares, negocios y con menos participación asistencia a congresos, conferencias, servicios o peregrinaciones como se presenta en la Figura No. 2.

Figura 2. Motivos de viaje del turista en agosto 2015

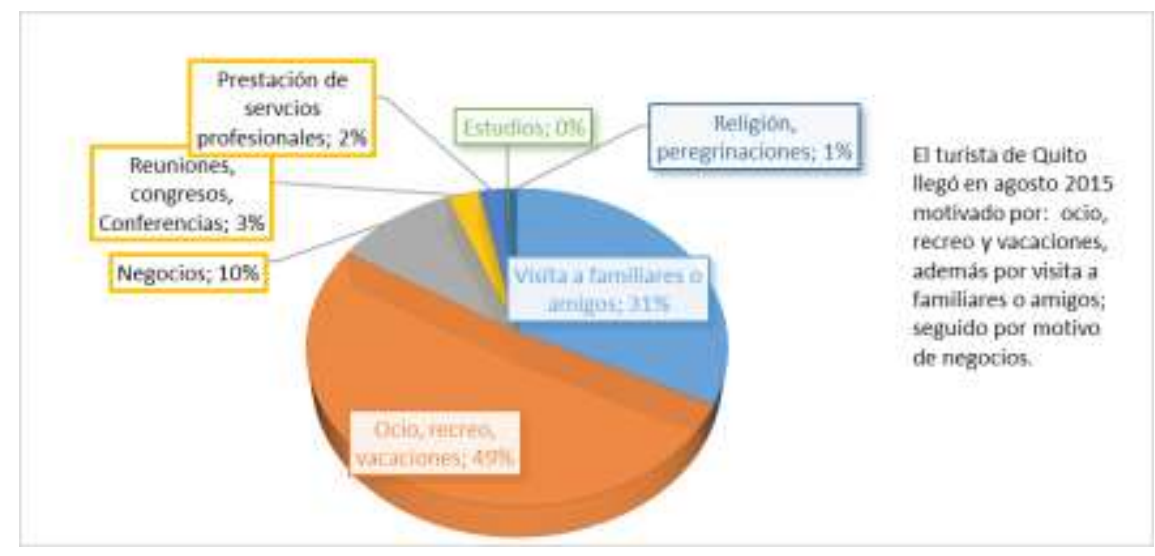

Fuente: (SIIT, 2019) 
Figura 3. Sitios más visitados por los turistas en Quito en el año 2016

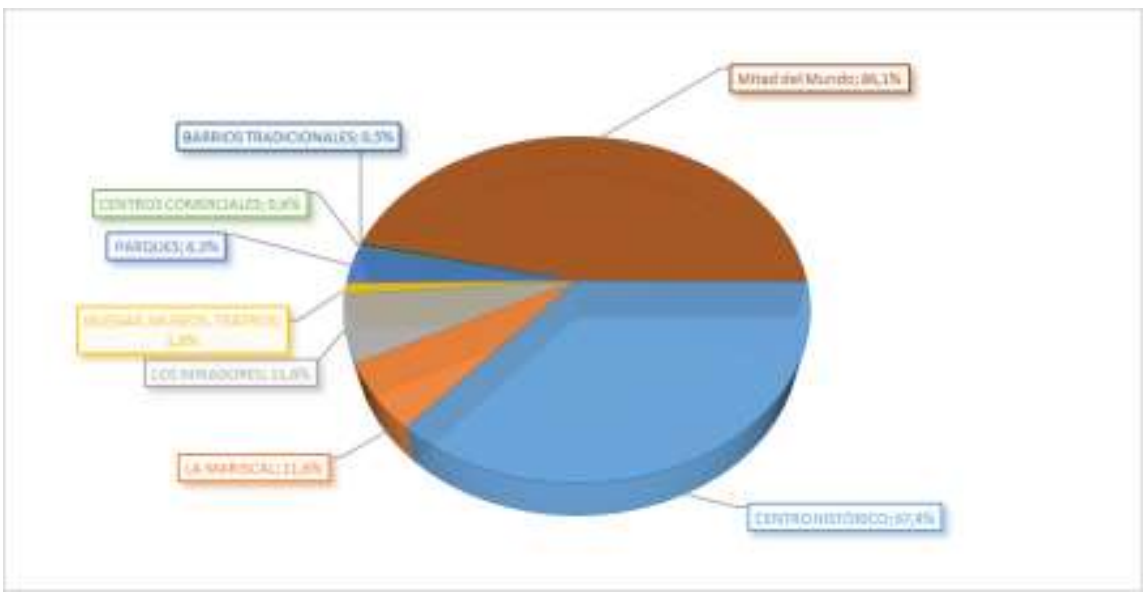

Fuente: (SIIT, 2019)

Al averiguar sobre los lugares que son visitados con mayor frecuencia por los turistas de Quito, destacan la mitad del mundo, el centro histórico, los miradores, la zona de "la mariscal”, parques, iglesias entre otros como se observa en la Figura No.3.

\section{Aspectos actuales de los mercados municipales de Quito}

Definitivamente los mercados representan una manifestación de cultural de las ciudades. Landeta (2014), afirma que "en los mercados de Quito se puede ver la diversidad de productos y culturas que hay en la ciudad". Precisamente, en los mercados municipales de la capital se puede encontrar una serie de productos que va desde alimentos frescos, gastronomía tradicional y actual, bebidas, flores, hasta hierbas que pueden aportar a la mejora de la salud o del amor. En los comedores de estos típicos lugares, se ofrece al público una gama de opciones acompañada de un trato personalizado con frases como: "venga mi bonito, mi reycito, mi lindito", entre otras que captan la atención de quienes llegan en busca de satisfacer su paladar. (Landeta, 2014)

Dentro de la urbe capitalina, los mercados también se destacan como atractivos turísticos, así es el caso del mercado de San Francisco, que data de hace más de 120 años y en donde existe una variedad de "plantas medicinales, como así también frutas, artesanías y comidas regionales" (Macipe, s.f.), En la Figura No.4 se puede evidenciar la variedad de artículos que se pueden hallar en este mercado del centro histórico. 
Figura 4. Imagen de una vendedora del mercado San Francisco

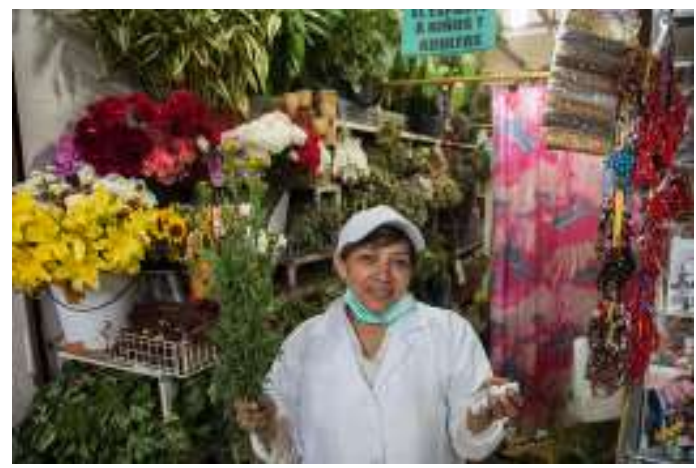

Fuente: (Macipe, s.f.)

Estos espacios de comercio minoritario, sobre todo los que se sitúan en el centro histórico, forman parte de los atractivos culturales para los turistas que llegan a la ciudad. Sin embargo, estos espacios requieren pulir su organización interna para poder atender de mejor manera a los visitantes nacionales o extranjeros. En referencia a este tema, un estudio a uno de los mercados del centro histórico, Suárez, Huaraca y Paladines (2017) determinaron que "Los trabajadores del mercado reconocen las dificultades actuales que presentan y expresan expectativas para su solución y crecimiento desde lo económico y social...” (pág. 338).

Al respecto se puede decir que el Municipio de Quito está trabajando, desde hace unos años, en algunos puntos que podrían considerarse como modernización de los mercados. Es así que por ejemplo, desde el año 2017 se procedió con la iniciativa de "Mercados Saludables" en la que se incluyó a varios de estos espacios, para hacer un diagnóstico de la situación actual y, luego de efectuar un trabajo conjunto con personas de la Secretaría de Salud, los comerciantes reciben el certificado que valida 133 puntos para controlar el higiene y buenas prácticas que benefician a clientes así como a los mismos vendedores (Municipio de Quito, 2017).

Otro elemento que es parte de este proceso de cambio de los mercados municipales, es el nivel de seguridad que tienen. Como una medida de apoyo, la Secretaría de Seguridad del Municipio de Quito, está en un proyecto de instalación de botones de pánico en los mercados, para que tanto clientes como comerciantes puedan activarlos ante cualquier incidente que pudiera suceder y así tener la visita de agentes de seguridad de la policía. (Romero D. , 2018)

\section{Gastronomía}

La gastronomía ancestral también puede considerarse parte de la identidad cultural transmitida de generación en generación en Quito. Es así que existen todavía algunos alimentos prehispánicos como la humita, que es preparada en base de choclo, el ají que fue consumido en la dieta de los quitus, o las tortillas de maíz conocidas hasta la actualidad como de tiesto (El Telégrafo, 2014). Todos estos alimentos forman parte viva de las raíces de los pueblos que habitaron en el territorio ecuatoriano hace cientos de años. 
Entre la gastronomía ancestral que se puede encontrar en Quito, consta la "tradicional colada morada" comúnmente preparada y degustada en noviembre por el día de los difuntos, que entre sus principales ingredientes integra al mortiño, fruto tradicional del Ecuador (Coba, y otros, 2012). Otro platillo de temporada es la fanesca, que usualmente es preparado y consumido en la semana santa y, si bien tiene una influencia hispánica, posee sus orígenes en el ritual agrario de hace cientos de años, en el que se agradecía al dios sol por las cosechas recibidas (Madrid \& Bedoya, 2013).

La "carita de Dios" tiene también cualidades gastronómicas actuales; es así que, entre sus principales platillos típicos buscados por turistas, se incluyen al chancho hornado, la tradicional fritada de cerdo, la guatita, que a pesar de ser un platillo que proviene de la costa ecuatoriana se encuentra regularmente en la capital. También son platillos tradicionales el Yahuarlocro, las tripas mishqui, papas con cuero, empanadas de viento, entre otros que se encuentran en los distintos comedores de diferente estrato de la capital. (El Telégrafo, 2016)

La gastronomía de Quito es variadísima, no solo por sus platillos tradicionales, ancestrales, sino también por una amplia gama de preparaciones modernas que han llegado desde todos los rincones del planeta, ya que en la urbe capitalina se pueden hallar comedores y comidas de sabores sudamericanos, norteamericanos, europeos o asiáticos. Sin embargo, Unigarro (2015) afirma que el "...patrimonio alimentario... busca ser protegido, ante la amenaza de su homogenización..."; sin duda una preocupante situación que refuerza la necesidad de generar una revitalización de la identidad cultural y alimenticia de los capitalinos.

\section{Expectativa del turismo en Quito}

De acuerdo a información publicada por el Municipio de Quito (QuitoInforma, 2019), en este año la Alcaldía efectuó promoción de la ciudad con una campaña que lleva el eslogan "Tu Historia Comienza en Quito", por medio de su ente oficial Quito Turismo. Esta acción se efectuó en la Feria Internacional del Turismo (FITUR), cuya versión No. 39 se realizó en la ciudad de Madrid durante los últimos días del mes de enero de este año. La promoción incluyó la exposición de las actividades de aventura que se efectúan en los alrededores capitalinos, la gastronomía, arte y sobre todo cultura.

De acuerdo las proyecciones del Sistema Institucional de Indicadores Turísticos (SIIT, 2019), se espera un crecimiento del 3,3\% en la cantidad de turistas que llegarán a Quito para el año 2019 y, a partir del 2020, se proyecta 4\% de aumento para cada período. Estas cifras podrían incrementarse en el caso de que exista mayor promoción de la ciudad en el exterior.

\section{Discusión}

El turismo constituye una actividad en acelerado crecimiento durante los últimos años. En la actualidad se habla de diferentes clases de turismo, en el que destacan el de descanso, aventura, salud, cultura, entre otros. En el caso de los datos presentados respecto a Ecuador 
y la ciudad de Quito específicamente, se puede verificar un constante aumento de visitantes que, si bien no corresponde a grandes porcentajes para cada año, esto genera un impacto para la economía de la urbe capitalina.

Una parte importante del turismo en Quito corresponde al denominado cultural, si es que se considera que el $67 \%$ de visitantes que llegan a esta urbe, afirmaron frecuentar el centro histórico de la ciudad, en donde se ubican muchos de sus atractivos culturales en los que se incluyen los mercados municipales más antiguos.

Pastor (2003) afirma que la falta de conocimiento “...tanto del patrimonio en sí como de su valor como elemento identitario, es el mayor problema con el que nos enfrentamos a la hora de hablar del respeto a la cultura en el ámbito del turismo cultural". Esto conlleva el hecho de que se deben destacar y fortificar todos los elementos de la cultura citadina, especialmente aquellos que forman parte de nuestra idiosincrasia basada en las raíces que tenemos.

Toselli (2006), efectuó un análisis del turismo cultural desde varios enfoques, uno de ellos parte de la globalización, tendencia que ha incrementado notablemente en los últimos años las cifras de visitantes a nivel mundial; sin embargo, afirma que "por el lado de las culturas receptoras, la necesidad de redescubrir y fortalecer la identidad cultural, como así también, resignificar el patrimonio como factor de unidad."

Es imperante que las ciudades y países que reciben gran cantidad de visitantes, consideren este latente requerimiento de fortificar la identidad cultural. En ese contexto, para la ciudad de Quito, se debe tomar en cuenta la necesidad de fomentar las costumbres y tradiciones que conforman su patrimonio, no solamente a nivel de construcciones, gastronomía, arte, sino también en la manera de ser tan propia de sus habitantes.

Este refuerzo cultural es muy necesario en la actualidad, debido a la migración de cientos de miles de ciudadanos de otros países entre los que destacan Colombia, Cuba, Haití o Venezuela, que traen consigo sus propias características culturales y en algunos casos, a pesar de ser similares, impactan en el acervo local.

Respecto de este importante tema, Navarro y Vaca (2018) elaboraron un documental denominado "El sueño ecuatoriano", cuyo "... eje central fue la migración hacia el Ecuador por razones económicas, sociales y políticas, haciendo énfasis en la realidad que viven los migrantes en la ciudad de Quito". Los autores destacaron las necesidades reales de los migrantes de diferentes lugares y sus motivos para llegar a esta ciudad.

Dentro del contexto cultural quiteño, se pueden mencionar a los mercados municipales, no solo como actores donde se oferta una variada gastronomía, sino también como espacios en los que se exponen los saberes ancestrales que destacan la riqueza del conocimiento capitalino. $\mathrm{Y}$ es que, en estos lugares de comercio minoritario, se expenden una serie de 
bienes que datan de cientos de años, como son las hierbas que se ofertan para cualquier mal, o en las artesanías que todavía son elaboradas a mano.

Para Mckee y Arguello (1988), en lugar de que se considere a los mercados como espacios caóticos, se debería entender que, "cada aspecto responde a una racionalidad, determinada por la historia, la geografía, la comercialización, el grado de tecnificación y las costumbres" (pág. 185). En este sentido, los mercados municipales también tienen un orden interno que se ha adaptado a las cambiantes condiciones de los avances de la ciudad capitalina.

Uno de los atractivos de los mercados municipales de la urbe quiteña, es justamente su infraestructura, la personalización en la manera de atención de los comerciantes hacia sus clientes, la variedad de artículos que se pueden encontrar, el conocimiento que se puede hallar en cada uno de los puestos, sobre todo de aquellos en los que el expendedor lleva varios años o toda su vida y, generalmente sus antecesores también se dedicaron a ese tipo de actividad. Así también, la gastronomía que guarda sabores ancestrales y típicos de las calles capitalinas y de su gente, constituyen un atractivo cultural riquísimo para compartirlo con los visitantes que provienen del mundo entero.

\section{Conclusiones}

- El turismo representa una actividad en claro crecimiento para Ecuador y para la ciudad de Quito, debido a sus múltiples atractivos. De acuerdo a las cifras del Municipio de Quito sobre turismo, el aspecto colonial que destaca la urbe capitalina en su centro histórico, representa un atractivo que, junto a la mitad del mundo, atrapan la atención de quienes han llegado a la ciudad capital.

- Los mercados municipales son parte de cultura y la tradición quiteña, más allá de representar un espacio de comercio minoritario, estos lugares son también motivo de visita de locales y extranjeros, para consumir los productos que allí se expenden, y vivenciar cada uno de los aromas y sabores que se pueden encontrar en estos espacios. De acuerdo a datos recientes, el Municipio se encuentra en un esfuerzo por mejorar los mercados municipales quiteños, iniciando con los que han sido reportados por evidenciar falencias en ámbitos de seguridad o higiene.

- Los saberes ancestrales son un aspecto vital de nuestra cultura ecuatoriana y quiteña. Estos conocimientos están dejando de ser vistos como algo simple y anticuado que debe ser desechado. Este tipo de conocimiento no solo representa una tradición en la manera en cómo se hacen las cosas, sino también son una expresión de la forma de vida de los antepasados que habitaron esta geografía.

- La gastronomía incluye también aquellos platillos, sabores y productos ancestrales, que son un atractivo para locales y visitantes que llegan a la urbe en busca de nuevas experiencias culturales. 


\section{Recomendaciones}

- Es imperante que las autoridades fomenten la expresión cultural del pueblo quiteño, y esto resalte con mayor fuerza la cultura como un atractivo de turismo. Sin embargo, es necesario que quienes lideran, destaquen la necesidad de mantener los rasgos culturales de la urbe quiteña, como un tesoro que se puede compartir con el resto de la humanidad, pero que no se debe difuminar con las diferentes tradiciones de otros pueblos, cuya gente se ha integrado a la capital en años recientes.

- Si bien la municipalidad se encuentra en proceso para brindar herramientas que mejoren los mercados, es responsabilidad de quienes comercian, el organizarse y apoyar un mejoramiento para la ejecución de su actividad, así como para la comodidad, higiene y seguridad de los clientes; sin que estos cambios representen un impacto en los atractivos principales de los mercados.

- La capital del Ecuador es una fuente de saberes ancestrales que se expresan en su forma cotidiana de vida. Lejos de que la global tendencia tecnológica los anule, debe implementarse estrategias para que estos conocimientos tradicionales cobren vida en cada uno de los capitalinos, y se constituyan en fuentes de identidad que apoyen a una mejor vida en sociedad.

- Los mercados son sitios que ofertan una variedad gastronómica en la que se incluyen platillos ancestrales, pero también actuales; en ese contexto, no se debe perder la manera tradicional de prepararlos y ofrecerlos a los clientes, ya que esto también representa un atractivo cultural que genera turismo.

\section{Referencias bibliográficas.}

ACTIVATE. (2014). Saberes Ancestrales. Obtenido de Activate EC: http://www.activate.ec/content/saberes-ancestrales

Bernal, C. (2010). Metodología de la investigación: administración, economía, humanidades y ciencias sociales. Bogotá: Pearson.

Carvallo, N. (13 de Septiembre de 2015). Saberes ancestrales: lo que se sabe y se siente desde siempre. Obtenido de El Telégrafo: https://www.eltelegrafo.com.ec/noticias/buen/1/saberes-ancestrales-lo-que-se-sabey-se-siente-desde-siempre

Coba, P., Coronel, D., Verdugo, K., Paredes, M., Yugsi, E., \& Huachi, L. (2012). ESTUDIO ETNOBOTÁNICO DEL MORTIÑO (Vaccinium floribundum) COMO ALIMENTO ANCESTRAL Y POTENCIAL ALIMENTO FUNCIONAL. La Granja, 5-13.

El Telégrafo. (25 de Mayo de 2014). El sabor de la cocina ancestral. Obtenido de El Telégrafo: https://www.eltelegrafo.com.ec/noticias/quito/1/el-sabor-de-la-cocinaancestral 
El Telégrafo. (14 de Octubre de 2016). Los 10 platos típicos de Quito que no puede perderse. Obtenido de El Telégrafo: https://www.eltelegrafo.com.ec/noticias/quito/1/10platillos-tipicos-que-fascinaran-a-los-visitantes-del-habitat-iii

Guerrero, K. (2015). MERCADOS EMBLEMÁTICOS DEL D. M. DE QUITO Y SU PATRIMONIO ALIMENTARIO: EL CASO DEL MERCADO SANTA CLARA E ITINERARIO TURÍSTICO CULTURAL DE LA COMUNA SANTA CLARA DE SAN MILLÁN. Quito: Uninversidad Central del Ecuador.

Landeta, D. (26 de Junio de 2014). Recorriendo los mercados de Quito. Obtenido de El Comercio: $\quad$ https://www.elcomercio.com/tendencias/recorriendo-mercadosquito.html

Macipe, A. (s.f.). 10 atractivos turísticos para visitar en Quito. Obtenido de National Geographic en español: https://www.ngenespanol.com/lugares/10-atractivosturisticos-visitar-quito/

Madrid, D., \& Bedoya, M. (2013). Memoria, oralidad y narrativa como recursos comunicacionales de la tradición popular de la fanesca en la Comunidad de Tolóntag (parroquia Píntag). Quito: Universidad Central del Ecuador.

Martín, V. (2010). Mercados municipales: la respuesta de los consumidores. Distribución y Consumo, 15-28.

Mckee, L., \& Arguello, S. (1988). Nuevas investigaciones antropológicas ecuatorianas. Quito: Abya-Yala.

Municipio de Quito. (1 de Diciembre de 2017). Municipio de Quito aplica la estrategia "Mercados Saludables". Obtenido de Quito Informa: http://www.quitoinforma.gob.ec/2017/12/01/municipio-de-quito-aplica-laestrategia-mercados-saludables/

Navarro, J., \& Vaca, D. (2018). Documental acerca de los migrantes extranjeros que habitan en la ciudad de Quito. Quito: Universidad Politécnica Salesiana.

OMT. (2019). Panorama OMT del turismo internacional edición 2018. Madrid: OMT.

Pastor, M. (2003). El patrimonio cultural como opción turística. Horizontes Antropológicos.

Pérez, R. (18 de Agosto de 2018). Saberes ancestrales, patrimonios ocultos. Obtenido de La Razón: http://www.la-razon.com/opinion/columnistas/Saberes-ancestralespatrimonios-ocultos_0_2985301500.html

QuitoInforma. (21 de Enero de 2019). Quito promocionará su oferta turística en Fitur 2019. Obtenido de portal web del Municipio de Quito: 
http://www.quitoinforma.gob.ec/2019/01/21/quito-promocionara-su-oferta-turisticaen-madrid/

Ramírez, M. (2011). Investigación cualitativa: definición y diseño. En E. Lucio-Villegas, Investigación y práctica en la educación de personas adultas (págs. 31-40). Valencia: Nau Llivres.

Rodríguez, E. (2005). Metodología de la Investigación. Villahermosa: Universidad Juárez Autónoma de Tabasco.

Romero, D. (27 de Febrero de 2018). 17 mercados de Quito tienen botones de pánico. Obtenido de El Comercio: https://www.elcomercio.com/actualidad/mercados-quitobotonesdepanico-seguridad-proyecto.html

Romero, E. (8 de Septiembre de 2018). Quito, la primera ciudad del mundo en ser declarada Patrimonio Cultural de la Humanidad. Obtenido de RT: https://actualidad.rt.com/actualidad/287934-ecuador-quito-cuarenta-aniversariopatrimonio-humanidad

SIIT. (2019). Sistema Institucional de Indicadores Turísticos del Municipio de Quito. Quito: Empresa Pública Metropolitana de Gestión de Destino Turístico.

Suárez, J., Huaraca, L., \& Paladines, G. (2017). Desarrollo turístico de los mercados municipales en centros históricos. Mercado San Francisco. Quito-Ecuador. Publicando, 331-339.

Toselli, C. (2006). Algunas reflexiones sobre el turismo cultural. PASOS: revista de turismo y patrimonio cultural, 175-182.

Unigarro, C. (2015). Sistemas alimentarios y patrimonio alimentario.Transculturaciones en el caso ecuatoriano. Cuadernos de Antropología, 21-34.

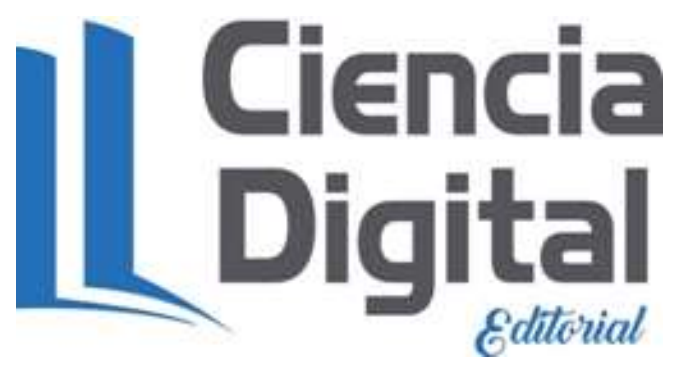




\section{Para citar el artículo indexado.}

Suárez J., Paladines G., Echarri M., \& Salinas E. (2019). Mercados municipales, escenarios de saberes ancestrales y turismo en la ciudad de Quito-Ecuador. Revista electrónica Ciencia Digital 3(2), 60-76. Recuperado desde: http://cienciadigital.org/revistacienciadigital2/index.php/CienciaDigital/article/view/375/820

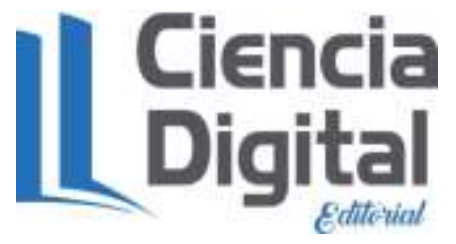

El artículo que se publica es de exclusiva responsabilidad de los autores y no necesariamente reflejan el pensamiento de la Revista Ciencia Digital.

El articulo queda en propiedad de la revista y, por tanto, su publicación parcial y/o total en otro medio tiene que ser autorizado por el director de la Revista Ciencia Digital.
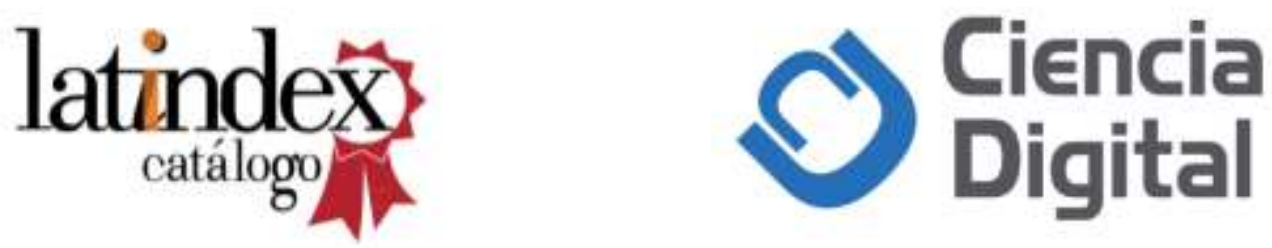